\title{
FRICTIONAL CONTACT PERFORMANCE TEST AND ANALYSIS OF CIRCULAR-ARC BIONIC NON-SMOOTH CONCAVE SURFACE
}

\author{
Sui Xiuhua ${ }^{1}$, Liu Cui ${ }^{2}, \mathrm{Su} \mathrm{Xu}^{3}$, He Jing ${ }^{4}$ \\ ${ }^{I}$ College of Mechanical and Electronic Engineering, Shandong University of Science and Technology, Qingdao, \\ 266590, China \\ ${ }^{2}$ College of Mechanical and Electronic Engineering, Shandong University of Science and Technology, Qingdao, \\ 266590, China \\ ${ }^{3}$ College of Mechanical and Electronic Engineering, Shandong University of Science and Technology, Qingdao, \\ 266590, China \\ ${ }^{4}$ College of Mechanical and Electronic Engineering, Shandong University of Science and Technology, Qingdao, \\ 266590, China
}

\begin{abstract}
Circular arc bionic non-smooth concave surface is used in friction driving. The frictional contact performance between circular arc bionic non-smooth concave surface and contact area is critical role for the mechanical drive of equipment whole. In this paper through orthogonal polynomial regression experiments, the influence of diameter, distribution density and height of the coating layer unit of circular arc non-smooth bionic concave on the friction coefficient is studied. The regression equation between the non-smooth geometric parameters of the circular arc bionic non-smooth concave surface and the frictional coefficient is obtained by experiment, and get the optimal solution by optimization analysis of regression equation. The experiment result shows that circular arc bionic non-smooth concave surface can increase the driving force and improve the transmission efficiency
\end{abstract}

Keywords: frictional contact, optimization design, bionic, concave surface $* * *$

\section{INTRODUCTION}

Friction is relative motion between two surfaces. One surface is positive and the other is passive. Generally, the passive surface is objective existence, which has two common types, flexible surface and rigid surface.

Many experts and scholars devoted to the research of friction and wear for years. In recent years a lot of researches, such as anti-friction and reducing adhesion and resistance, were conducted by using the bionic technology [1-4]. But the study of frictional contact performance of circular arc bionic non-smooth concave surface is very rare.

In this paper, circular arc bionic non-smooth concave surface is regard as positive, the influence of diameter, distribution density and height of the concave on the friction coefficient of this surface is studied, through orthogonal polynomial regression experiments, also is obtained the regression equation between the non-smooth geometric parameters of the concave surface and the frictional coefficient, and get the optimal solution by optimization analysis of regression equation.

\section{DESIGN OF THE EXPERIMENTAL PROGRAM}

Orthogonal polynomial regression experiments are designed to obtain approximate function relation between the experimental results and the experimental factors. Circular arc bionic non-smooth concave surface wrapped in normal driving roller as covering layer, which served as positive surface, and the passive surface is rubber belt. The influence of diameter and distribution density of concave on the friction coefficient is studied through the critical sliding friction test method.

The test indicator is friction coefficient $y$, which influenced by three main factors: the height of the spherical cap of nonsmooth unit $x_{l}(h)$; projected diameter of the spherical cap of non-smooth unit $x_{2}(b)$; adjacent non-smooth unit center distance $x_{3}(s)$. M represents the number of test factors, namely $\mathrm{m}=3[5-10]$.

\subsection{Determine the Encode Table of the}

\section{Experimental Factors}

Firstly, determine the range of factors $\left(\mathrm{x}_{\mathrm{j}} ; \mathrm{j}=1,2 \ldots \mathrm{m}\right)$ and the number of zero level test $\left(\mathrm{m}_{0}\right)$. The asterisk arm length must satisfy the relational expression 2.1 based on the orthogonal requirements.

$$
r=\sqrt{\frac{-m_{c}+\sqrt{\left(m_{c}+2 m+m_{0}\right) m_{c}}}{2}}
$$


Where, $m_{c}$, the number of second level test, $m_{c}=8 ; 2 m$ is the number of asterisk trial, $2 m=6 ; m_{0}$ the number of zero level test, $m_{0}=1$.

So look-up table can get $\mathrm{r}^{2}=1.476, \mathrm{r}=1.215$.

Then to determine the changes in spacing of experimental factors is shown as the formula 2.2:

$$
\Delta_{j}=\frac{x_{j \gamma}+x_{j 0}}{\gamma}
$$

Next encoded factor levels get standardized variable $\mathrm{z}_{\mathrm{j}}(\mathrm{j}=1,2, \ldots, \mathrm{m})$ is shown in 2.3.Code is the linear transformation of each levels of $x_{j}$.

$$
z_{j}=\frac{x_{j \gamma}+x_{j 0}}{\Delta_{j}}
$$

Where $z_{j}$ is the encoding of the test factor $x_{j}$, they are oneto-one correspondence, so the value of specification variable $\left(z_{j}\right)$ is not influenced by units and size of natural variables $\left(\mathrm{x}_{\mathrm{j}}\right)$.Code can convert regression problem between the test results $\mathrm{y}$ and the level of factors $\mathrm{x}_{\mathrm{j}}$ into regression problem

between the test results $y$ and coded values $z_{\mathrm{j}}$. After coding, the level of the experimental factors are numbered- $\gamma, 1,0,1, \gamma$.

According to the basic principles and the main equations of the quadratic regression orthogonal design, determine the coding table of $z_{1}, z_{2}, z_{3}$ under previous test results and the actual situation, as shown in Table-1.

Table-1 Encode table of the experimental factors

\begin{tabular}{|l|l|l|l|}
\hline \multirow{2}{*}{ standardized variable $\mathrm{z}_{\mathrm{j}}$} & \multicolumn{3}{|c|}{ natural variables $\left(\mathrm{x}_{\mathrm{j}}\right)$} \\
\cline { 2 - 4 } & $\mathrm{x}_{1}$ & $\mathrm{x}_{2}$ & $\mathrm{x}_{3}$ \\
\hline An asterisk arm $\gamma$ & 7.43 & 74.3 & 210.75 \\
\hline Upper lever 1 & 7.00 & 70.0 & 200.00 \\
\hline
\end{tabular}

\begin{tabular}{|l|l|l|l|}
\hline Zero lever 0 & 5.00 & 50.0 & 150.00 \\
\hline Lower lever-1 & 3.00 & 30.0 & 100.00 \\
\hline Lower asterisk arm $-\gamma$ & 2.57 & 25.7 & 89.25 \\
\hline Change interval $\Delta \mathrm{j}$ & 2.00 & 20.0 & 50.00 \\
\hline
\end{tabular}

\subsection{Ternary Quadratic Regression Orthogonal}

\section{Design}

Orthogonal design consists of three test points, second level test $\mathrm{m}_{\mathrm{c}}\left(\mathrm{m}_{\mathrm{c}}=8\right)$, asterisk test $2 \mathrm{~m}(2 \mathrm{~m}=6)$ and zero-level test $\mathrm{m}_{0} \quad\left(\mathrm{~m}_{0}=1\right)$. Therefore the test total number of ternary quadratic regression is determined by the formula 2.4 .

$$
N=m_{c}+2 m+m_{0}=8+6+1=15
$$

The experimental project of ternary quadratic regression orthogonal design is shown in the Table -2

Table-2 Experimental project table

\begin{tabular}{|l|l|l|l|l|}
\hline number & $Z_{1}$ & $Z_{2}$ & $Z_{3}$ & $y$ \\
\hline 1 & 1 & 1 & 1 & $y_{1}$ \\
\hline 2 & 1 & 1 & -1 & $y_{2}$ \\
\hline 3 & 1 & -1 & 1 & $y_{3}$ \\
\hline 4 & 1 & -1 & -1 & $y_{4}$ \\
\hline 5 & -1 & 1 & 1 & $y_{5}$ \\
\hline 6 & -1 & 1 & -1 & $y_{6}$ \\
\hline 7 & -1 & -1 & 1 & $y_{7}$ \\
\hline 8 & -1 & -1 & -1 & $y_{8}$ \\
\hline 9 & $\gamma$ & 0 & 0 & $y_{9}$ \\
\hline 10 & $-\gamma$ & 0 & 0 & $y_{10}$ \\
\hline 11 & 0 & $\gamma$ & 0 & $y_{11}$ \\
\hline 12 & 0 & $-\gamma$ & 0 & $y_{12}$ \\
\hline 13 & 0 & 0 & $\gamma$ & $y_{13}$ \\
\hline 14 & 0 & 0 & $-\gamma$ & $y_{14}$ \\
\hline 15 & 0 & 0 & 0 & $y_{15}$ \\
\hline
\end{tabular}

For the ternary quadratic regression orthogonal design, orthogonal design coding table is obtained by using the secondary center of methods, shown in Table-3

Table-3 Encode table of orthogonal design

\begin{tabular}{|l|l|l|l|l|l|l|l|l|l|}
\hline Test number & $z_{1}$ & $z_{2}$ & $z_{3}$ & $z_{1} z_{2}$ & $z_{1} z_{3}$ & $z_{2} z_{3}$ & $z_{1}{ }^{\prime}$ & $z_{2}{ }^{\prime}$ & $z_{3}{ }^{\prime}$ \\
\hline 1 & 1 & 1 & 1 & 1 & 1 & 1 & 0.270 & 0.270 & 0.270 \\
\hline 2 & 1 & 1 & -1 & 1 & -1 & -1 & 0.270 & 0.270 & 0.270 \\
\hline 3 & 1 & -1 & 1 & -1 & 1 & -1 & 0.270 & 0.270 & 0.270 \\
\hline 4 & 1 & -1 & -1 & -1 & -1 & 1 & 0.270 & 0.270 & 0.270 \\
\hline 5 & -1 & 1 & 1 & -1 & -1 & 1 & 0.270 & 0.270 & 0.270 \\
\hline 6 & -1 & 1 & -1 & -1 & 1 & -1 & 0.270 & 0.270 & 0.270 \\
\hline 7 & -1 & -1 & 1 & 1 & -1 & -1 & 0.270 & 0.270 & 0.270 \\
\hline 8 & -1 & -1 & -1 & 1 & 1 & 1 & 0.270 & 0.270 & 0.270 \\
\hline 9 & 1.215 & 0 & 0 & 0 & 0 & 0 & 0.747 & -0.730 & -0.730 \\
\hline 10 & -1.215 & 0 & 0 & 0 & 0 & 0 & 0.747 & -0.730 & -0.730 \\
\hline 11 & 0 & 1.215 & 0 & 0 & 0 & 0 & -0.730 & 0.747 & -0.730 \\
\hline
\end{tabular}




\begin{tabular}{|l|l|l|l|l|l|l|l|l|l|}
\hline 12 & 0 & -1.215 & 0 & 0 & 0 & 0 & -0.730 & 0.747 & -0.730 \\
\hline 13 & 0 & 0 & 1.215 & 0 & 0 & 0 & -0.730 & -0.730 & 0.747 \\
\hline 14 & 0 & 0 & -1.215 & 0 & 0 & 0 & -0.730 & -0.730 & 0.747 \\
\hline 15 & 0 & 0 & 0 & 0 & 0 & 0 & -0.730 & -0.730 & -0.730 \\
\hline
\end{tabular}

\subsection{Determining and Implementing Testing Scheme}

According to the testing scheme which determined by ternary quadratic regression orthogonal design table, the 15 experiments is carried out and 15 test indicators is obtained. Table- 4 is statistical analytical table of orthogonal design. In this table:

$$
\begin{aligned}
& D_{j}=\sum_{i=1}^{15} Z_{j i}^{2}, B_{j}=\sum_{i=1}^{15} Z_{j i}^{2} y_{i}, \\
& b=B_{j}+D_{j}, S_{j}=b_{j} B_{j}
\end{aligned}
$$

2.6 is the quadratic central processing formula:

$$
Z_{i j}^{\prime}=Z_{i j}^{2}-\frac{1}{N} \sum_{i=1}^{N} Z_{i j}^{2}=Z_{i j}^{2}-0.73
$$

The regression equation between geometric parameters (experimental factors) of bionic non-smooth surface and test indicator y is

\begin{tabular}{|c|c|c|c|c|c|c|c|c|c|c|c|c|}
\hline $\begin{array}{l}\text { Test } \\
\text { number }\end{array}$ & $z_{0}$ & $z_{1}$ & $z_{2}$ & $z_{3}$ & $z_{1} z_{2}$ & $z_{1} z_{3}$ & $z_{2} z_{3}$ & $z_{1}$ & $z_{2}^{\prime}$ & $z_{3}$ & $y_{i}$ & $y_{i}^{2}$ \\
\hline 1 & 1 & 1 & 1 & 1 & 1 & 1 & 1 & 0.270 & 0.270 & 0.270 & 0.44 & 0.1936 \\
\hline 2 & 1 & 1 & 1 & -1 & 1 & -1 & -1 & 0.270 & 0.270 & 0.270 & 0.32 & 0.1024 \\
\hline 3 & 1 & 1 & -1 & 1 & -1 & 1 & -1 & 0.270 & 0.270 & 0.270 & 0.37 & 0.1369 \\
\hline 4 & 1 & 1 & -1 & -1 & -1 & -1 & 1 & 0.270 & 0.270 & 0.270 & 0.38 & 0.1444 \\
\hline 5 & 1 & -1 & 1 & 1 & -1 & -1 & 1 & 0.270 & 0.270 & 0.270 & 0.45 & 0.2025 \\
\hline 6 & 1 & -1 & 1 & -1 & -1 & 1 & -1 & 0.270 & 0.270 & 0.270 & 0.3 & 0.09 \\
\hline 7 & 1 & -1 & -1 & 1 & 1 & -1 & -1 & 0.270 & 0.270 & 0.270 & 0.36 & 0.1296 \\
\hline 8 & 1 & -1 & -1 & -1 & 1 & 1 & 1 & 0.270 & 0.270 & 0.270 & 0.39 & 0.1521 \\
\hline 9 & 1 & 1.215 & 0 & 0 & 0 & 0 & 0 & 0.747 & $\overline{0} .730$ & $\overline{-} \mathbf{0 . 7 3 0}$ & 0.46 & 0.2116 \\
\hline 10 & 1 & $\overline{1.215}$ & 0 & 0 & 0 & 0 & 0 & 0.747 & 0.730 & 0.730 & 0.38 & 0.1444 \\
\hline 11 & 1 & 0 & 1.215 & 0 & 0 & 0 & 0 & $\overline{0} .730$ & 0.747 & $\overline{-} \mathbf{0} 730$ & 0.36 & 0.1296 \\
\hline 12 & 1 & 0 & -1.215 & 0 & 0 & 0 & 0 & 0.730 & 0.747 & 0.730 & 0.42 & 0.1764 \\
\hline 13 & 1 & 0 & 0 & 1.215 & 0 & 0 & 0 & $\overline{0}-\overline{730}$ & $\overline{0}-\overline{730}$ & 0.747 & 0.38 & 0.1444 \\
\hline 14 & 1 & 0 & 0 & $\overline{1.215}$ & 0 & 0 & 0 & $\overline{-}-730$ & $\overline{-}-730$ & 0.747 & 0.34 & 0.1156 \\
\hline 15 & 1 & 0 & 0 & 0 & 0 & 0 & 0 & $\overline{0} \mathbf{0} 730$ & $\overline{0}-730$ & $\overline{-}-730$ & 0.48 & 0.2304 \\
\hline$D_{j}$ & 15 & 10.95 & 10.95 & 10.95 & 8 & 8 & 8 & 4.365 & 4.365 & 4.365 & 5.83 & 2.304 \\
\hline $\mathrm{B}_{\mathrm{j}}$ & 5.83 & 0.107 & -0.063 & 0.279 & 0.01 & -0.01 & 0.31 & $\overline{0}-005$ & $-\overline{0} 049$ & $\overline{0}-182$ & & \\
\hline$b_{j}$ & 0.389 & 0.01 & -0.006 & 0.025 & 0.001 & 0.001 & 0.04 & $\overline{0} 0.001$ & $\overline{0} 0.021$ & $\overline{0} .042$ & & \\
\hline $\mathrm{s}$ & 2.266 & 0.001 & 0.0004 & 0.007 & 0 & 0 & 0.012 & 0 & 0.002 & 0.008 & & \\
\hline
\end{tabular}
got by the regression coefficient $b_{j}$ calculated from Table- 4 and removed not significant items

Table-4 Statistical analytical table of orthogonal design 


\subsection{Establish the Regression Equation}

The ternary quadratic regression equation of three experimental factors and test indicators $y$;

$$
\begin{aligned}
& y=a+b_{1} z_{1}+b_{2} z_{2}+b_{3} z_{3}+b_{12} z_{1} z_{2}+b_{23} z_{2} z_{3} \\
& +b_{13} z_{1} z_{3}+b_{11} z_{1}^{\prime}+b_{22} z_{2}^{\prime}+b_{33} z_{3}^{\prime}
\end{aligned}
$$

The regression coefficient is calculated, a regression equation with standardized variables is established. The calculation formula of regression coefficient as follows:

$$
\begin{aligned}
& a=\frac{1}{n} \sum_{i=1}^{n} y_{i}=\bar{y} \\
& b_{j}=\frac{\sum_{i=1}^{n} z_{j i} y_{i}}{\sum_{i=1}^{n} z_{j i}^{2}}, j=1,2, \ldots, m \\
& b_{k j}=\frac{\sum_{i=1}^{n}\left(z_{k} z_{j}\right)_{i} y_{i}}{\sum_{i=1}^{n}\left(z_{k} z_{j}\right)_{i}^{2}}, j>k, k=1,2, \ldots, m-1 \\
& b_{j j}=\frac{\sum_{i=1}^{n} z_{j i}^{\prime} y_{i}}{\sum_{i=1}^{n}\left(z_{j i}^{\prime}\right)^{2}}
\end{aligned}
$$

From table 6.4, $a=0.389, b_{1}=0.01, b_{2}=-0.006, b_{3}=0.025$, $\mathrm{b}_{12}=0.001, \mathrm{~b}_{13}=-0.001, \mathrm{~b}_{23}=0.04, \mathrm{~b}_{11}=-0.001, \mathrm{~b}_{22}=-0.021$, $\mathrm{b}_{33}=-0.042$

Then remove not significant items and get regression equation such as shown in equation 2.12 between geometric parameters of bionic non-smooth surface and frictional coefficient.

$$
\begin{aligned}
& y=0.389+0.025 z_{3}+ \\
& 0.04 z_{2} z_{3}-0.042 z_{3}^{\prime}
\end{aligned}
$$

\subsection{Significance Test of the Regression Equation}

In significance test of the regression equation, the meaning of the parameters and formulas as follows:

The total sum of squares:

$$
\begin{aligned}
& S S_{T}=\sum_{i=1}^{n}\left(y_{i}-\bar{y}^{2}\right)^{2}= \\
& \sum_{i=1}^{n}\left(y_{i}\right)^{2}-\frac{1}{n}\left(\sum_{i=1}^{n} y_{i}\right)^{2}
\end{aligned}
$$

Degrees of freedom of the total sum of squares:

$$
d f_{T}=n-1
$$

Partial regression sum of squares of one degree term:

$$
S S_{j}=b_{j}^{2} \sum_{i=1}^{n} z_{j i}^{2}, j=1,2, \ldots, m
$$

Degrees of freedom of partial regression sum of squares of one degree term:

$$
d f_{\text {one deg reeterm }}=1
$$

Partial regression sum of squares of interaction term:

$$
\begin{gathered}
S S_{k j}=b_{k j}^{2} \sum_{i=1}^{n}\left(z_{k} z_{j}\right)_{i}^{2}, \\
j>k, k=1,2, \ldots, m-1
\end{gathered}
$$

Degrees of freedom of partial regression sum of squares of interaction term:

$$
d f_{\text {int } \text { ractionitem }}=1
$$

Partial regression sum of squares of quadratic term:

$$
S S_{j j}=b_{j j}^{2} \sum_{i=1}^{n}\left(z_{j i}^{\prime}\right)^{2}
$$

Degree of freedom of partial regression sum of squares of quadratic term:

$$
d f_{\text {quadraticterm }}=1
$$

Regression sum of squares:

$$
\begin{aligned}
& S S_{R}=\sum S S_{\text {onedeg reeterm }}+\sum S S_{\text {interactionitem }} \\
& +\sum S S_{\text {quadraticterm }}
\end{aligned}
$$

Degrees of freedom of regression sum of squares:

$$
\begin{aligned}
& d f_{R}=\sum d f_{\text {onedeg reeterm }}+\sum d f_{\text {interactionitem }} \\
& +\sum d f_{\text {quadraticterm }}
\end{aligned}
$$

Residual sum of squares:

$$
S S_{e}=S S_{T}-S S_{R}
$$

Degrees of freedom of residual sum of squares:

$$
d f_{e}=d f_{T}-d f_{R}(2.24)
$$


Inspection of the regression coefficients:

$$
\begin{gathered}
F_{j}=\frac{M S_{j}}{M S_{e}}=\frac{S S_{j}}{S S_{e} / d f_{e}} \\
F_{k j}=\frac{M S_{k j}}{M S_{e}}=\frac{S S_{k j}}{S S_{e} / d f_{e}}
\end{gathered}
$$

$$
F_{j j}=\frac{M S_{j j}}{M S_{e}}=\frac{S S_{j j}}{S S_{e} / d f_{e}}
$$

According to the above formulas, through calculation, significance test of regression equation results can got which is shown in Table-5

Table-5 Significance test result table of regression equation

\begin{tabular}{|c|c|c|c|c|c|}
\hline Differences in source & SS & $\mathrm{d} f$ & $M S$ & $F$ & Significance \\
\hline$z_{3}$ & 0.0070 & 1 & 0.00700 & 7.3 & Significant \\
\hline$z_{2} z_{3}$ & 0.0128 & 1 & 0.01280 & 13.4 & Significant \\
\hline$z_{3}^{\prime}$ & 0.0077 & 1 & 0.07700 & 8.1 & Significant \\
\hline regression & 0.0275 & 3 & & & \\
\hline Residual & 0.0105 & 11 & 0.00096 & & \\
\hline sum & 0.0380 & 14 & & & \\
\hline
\end{tabular}

As can be seen from Table 5, the significance level of the regression equation was 0.25 , and good fitting.

\subsection{Back Substitution of the Regression Equation}

According to the coding formula or secondary center formula convert the regression relationship between the $\mathrm{z}_{\mathrm{j}}$ and test indicators $y$ into the regression relationship between the natural variables $\mathrm{x}_{\mathrm{j}}$ and test indicator $\mathrm{y}$. The conversion relational expression shown in 2.28,2.29, 2.30.

$$
\begin{aligned}
z_{3}^{\prime}=z_{3}^{2}-\frac{1}{n} \sum z_{3}^{2} & =z_{3}^{2}-0.73 \\
z_{2} & =\frac{x_{2}-50}{20} \\
z_{3} & =\frac{x_{3}-150}{50}
\end{aligned}
$$

The relational formula between the coefficient of friction and each test factors is obtained after back substitution of the conversion as shown in equation 2.31 as follows:

$$
\begin{aligned}
& y=0.2667-0.006 x_{2}+0.0035 x_{3} \\
& +0.00004 x_{2} x_{3}-0.0000168 x_{3}^{2}
\end{aligned}
$$

\section{OPTIMIZATION ANALYSIS OF THE REGRESSION EQUATION}

If the multi-function $f(x, y, z)$ is continuous in a bounded closed interval D, there must have the minimum and maximum values in D. Therefore, compared function value of the multi-function $f(x, y, z)$ stagnation point in $\mathrm{D}$ with the maximum and minimum values on the boundary, the independent variables of the regression equation can be obtained the optimal value[11-16]. The specific method is as follows:

The optimal test conditions is calculated according to the extreme value necessary condition

$\frac{\partial y}{\partial x_{1}}=0, \frac{\partial y}{\partial x_{2}}=0, \frac{\partial y}{\partial x_{3}}=0$. Therefore solving derivative of $\mathrm{x}, \mathrm{y}$ in formula2.31,obtain equations 3.1.

The solution of equations can get when the formula (3.1) equals to zero:

$$
\begin{aligned}
& \left\{\begin{array}{l}
\frac{\partial y}{\partial x_{2}}=-0.006+0.00004 x_{3} \\
\frac{\partial y}{\partial x_{3}}=0.00354+0.00004 x_{2}-0.0000336 x_{3}
\end{array}\right. \\
& X_{2}=37.5, x_{3}=150
\end{aligned}
$$

Making the point $(37.5,150)$ which is the stagnation point of the original equation into the formula 2.31, and obtain: $\mathrm{y}=0.42$

After comparison with the boundary value, when $\mathrm{x}_{1}=5 \mathrm{~mm}$, $\mathrm{x}_{2}=50 \mathrm{~mm}, \mathrm{x}_{3}=150 \mathrm{~mm}$. $\mathrm{y}$ has the maximum value, and $\mathrm{y}_{\max }$ $=0.48$

\section{TEST FACTOR ANALYSIS}

The degree of influence of various factors on the size of the friction coefficient is determined by the coefficients size of the regression equation. The three-dimensional map and contour map expressed the regression equation, as shown in Figure 1 and Figure 2. 

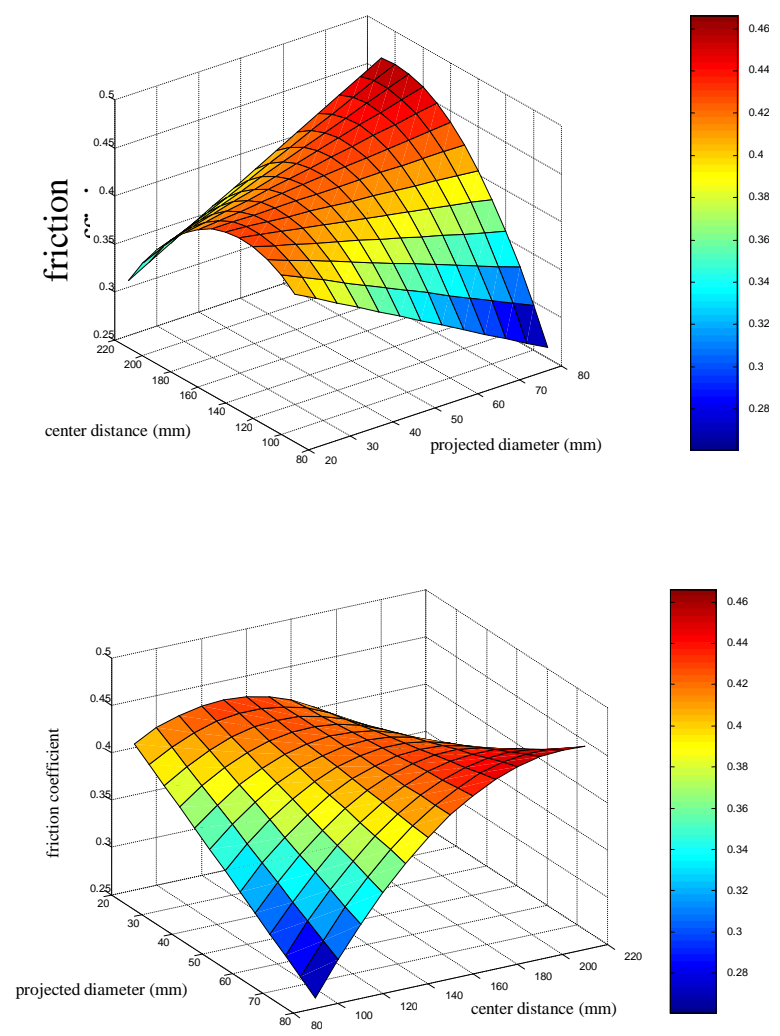

Fig-1 Effect of projected diameter and center distance on friction coefficient

Figure 1, which can see the trend of friction coefficient with the projector diameter and center distance, shows the relationship between projection diameter and center distance and the friction coefficient. Because the projection diameter and center distance on the friction coefficient is the process of interaction, the single factor graph is used in order to more accurately describe the impact of both on the friction coefficient, which are as shown in Figure 2 and Figure 3.

Figure 2 expresses the relationship between the center distance and the friction coefficient when the projected diameter is a fixed value. Figure 3 shows the relationship between the projected diameter and the friction coefficient when the center distance is a fixed value.

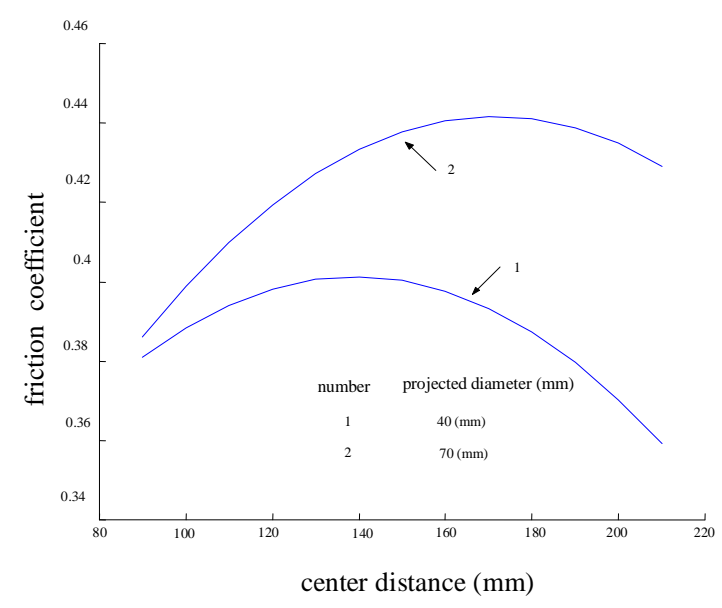

Fig-2 Relation between center distance and friction coefficient 


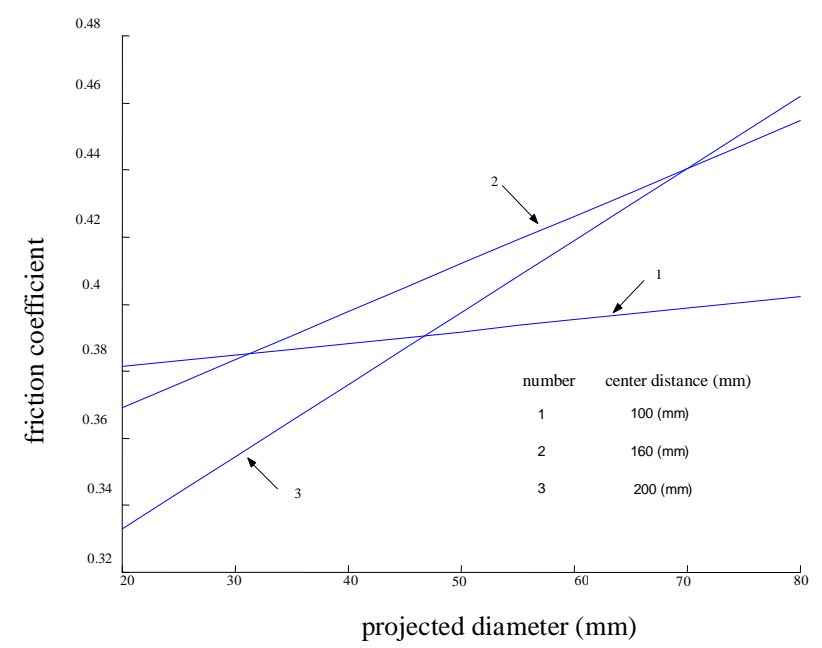

Fig-3 Relation between projected diameter and friction coefficient

As can be seen from Figure 2, center distance on the friction coefficient is not an independent role in the process, and the size of the center distance and projected diameter of spherical cap concave pit in the cladding layer have a certain relationship. When the spherical cap diameter is smaller, the friction coefficient curve is shown in curve 1. With the increase of the diameter of the spherical cap, as shown in curve 2 , the center distance value also increases when obtain the maximum coefficient of friction. However, they are not a linear proportional relationship, the friction coefficient showed a decreasing trend with further increase of the center distance.

Both the friction coefficient is also increased due to the increased contact area between the tape and circular arc bionic non-smooth concave surface which cladding layer material is rubber which belong to the super-elastic material. The increase in the projected diameter of the spherical cap also means that the increase of the contact area of the adhesive interface, so the friction coefficient increases. However, due to the band having a certain thickness, when the center distance of concave pit is small, the surface contact state of the cladding layer and belt is shown in Figure 4 that mutual contact area is reduced and leading to reduced friction coefficient. When the center distance is appropriate between two spherical cap concave surfaces, the belt and the coating layer surface contact state shows in Figure 5, and their mutual contact area and the friction coefficient increases. While when the center distance continues to increase, the contact area and the friction coefficient will decrease.

From Figure 3, when the value of center distance is small, the contact status of the tape with cladding layer of circular arc bionic non-smooth concave surface gradually shift from Figure 5 to Figure 4 with spherical cap concave pit diameter increases, and the friction coefficient decreases, which is shown in the curve 1 . When the center distance is larger, the contact of the tape cladding layer is gradually transformed into the state shown in Figure 5, as the concave pit diameter increases from $20 \mathrm{~mm}$ to $80 \mathrm{~mm}$, the friction coefficient gradually increases.
When the projector diameter and center distance are fixed in the test range, the spherical cap height increases, which means increasing the contact area of the tape and cladding layer, causing the friction coefficient increase. However, due to the increase of cap height leads to depression area between units increase and the contact state between the tape and the concave surface of the coating layer deteriorate, so balanced, the height of the spherical crown has little influence on the friction coefficient.

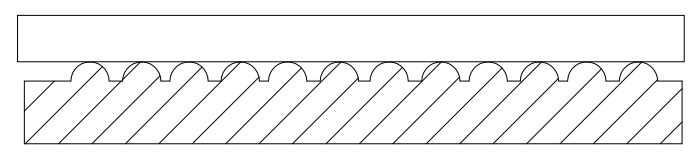

Fig-4 Contact state when the center distance is smaller

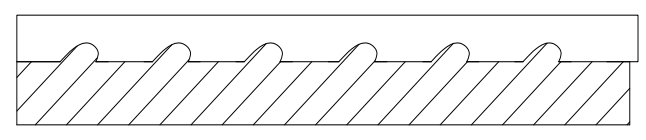

Fig-5 Contact state when the center distance is larger

\section{CONCLUSION}

(1) It is not an independent process that the friction coefficient is impacted by the center distance and the spherical cap projection diameter, but it is the result of the interaction between them.

(2) As long as the center distance, the spherical cap projection diameter and spherical cap height appropriate match, the effect of increasing friction which has the biomimetic coating layer is obvious.

\section{ACKNOWLEDGEMENTS}

We are very thankful to The Science and Technology Program of Shandong Province Higher Educational (Grant NO. J14LB12) and The Natural Science Foundation of Shandong Province (Grant No.ZR2012EEM004) for sponsoring to publish this paper. 


\section{REFERENCES}

[1]. Harrison A, Belt conveyor research 1980 2000, Bulk Solids Handling,2001 21(2).

[2]. Yang Dawen, Pang Yudong, Zhao Yushun, Zhao Jianping, Present situation and Prospect of belt conveyor of the domestic and international coal mine, Coal Mine Machinery,2002(1).

[3]. Xiao Linjing, Sui Xiuhua, Miao Dejun, Study on Mechanics of Driving Drum with Super-elastic convexity surface covering-layer structure, Joumal of bionic engineering(EI),2008.9.

[4]. Sui Xiuhua, Xiao Linjing, Miao Dejun, Finite Element Analysis on layer structure-covered concave sucker driving drum,2008(10).

[5]. Guo Yuhua, Shen Pengfei, Jin Hongbin, Zhao Kai, An Orthogonal Regression Experiment on Cold-Bonded Molding of Ore/Coal Composite Pellets, 2012, 24(3).

[6].Mao Shisong, Ding Yuan, Lv Naigang, Regression analysis and experiment design, Shanghai: East China Normal University Press, 1986.

[7] C. H. Chen, J. E. Zhou, The Orthogonal-Regression Analysis on Cooling Rate Of PVP Quenchant, Journal of Materials Engineering and Performance,2002,11(5).

[8]. Gao Yunyan, Orthogonal regression experiment design, Metallurgical Industry Press, 1988.

[9]. Shen Weiguo, Hu Jinqiang, Zhou Mingkai, Ma Wei, Cai Zhi, Application of Quadratic Orthogonal Regressive Analysis on the Three Ingredients Optimum Design of Cement, Journal of Wuhan University of Technology,2008 ,30(10).

[10]. Cheng Jia, Zhu Yu, Ji Linhong, Modeling Approach and Analysis of the Structural Parameters of an Inductively Coupled Plasma Etcher Based on a Regression Orthogonal Design, Plasma Science and Technology ,2012,14(12).

[11]. Yu Runwei, Zhu Xiaohui, Foundation and Application of MATLAB, Beijing Mechanical Industry Press, 2008.

[12]. Wang Zaizhou, Wang Zhongliang, The bionic nonsmooth shape simulation based on MATLAB, Lubrication and Sealing, 2006(8).

[13]. Alejandro C. Olivieri, Wu Hailong, Yu Ruqin, MVC3: A MATLAB graphical interface toolbox for third-order multivariate calibration, Chemometrics and Intelligent Laboratory Systems, 2012,116.

[14]. Tong Shuer, Wang Shengnan, Li Hainan, Geng Junhong, Flue-cured tobacco fertilization optimization scheme based on quadratic orthogonal regression test, Shan xi Agricultural Sciences,2011(4).

[15]. Zhou Xianhan, Jiang Kaiya, Optimization of the honey ferment sour milk by the regress-orthogonal design, Food science and technology,2007, 6 .

[16]. Guo Hongyuan, Hu Weisheng, Yu Youling, Design of advanced product line in automotive electronic product based on matlab graphical user interface, Journal of Computer Applications, 2011, 31 Suppl.2.

\section{BIOGRAPHIES}

Sui Xiuhua, associate professor, research major: mechanical and electronic engineering, email: suixh@126.com

Liu Cui, master degree student, email: liucui198904@163.com

Su Xu, master degree student, email: 450542509@qq.com

He Jing, master degree student, email: 1054463535@qq.com 